\title{
Predicting Ridership at railway intersection Station based on Accessibility Model
}

\author{
Caixia Han \\ School of Information technology, College of science \&Arts of Jianghan University.China
}

Keywords: Traffic engineering; railway intersection; ridership; accessibility; negative logistic function; GIS

\begin{abstract}
This paper explores a ridership forecasting method based on railway intersection site accessibility and vehicle use patterns. The accessibility model utilizes vehicle use and demographic data. In the study, we employ GIS to disaggregate population data into small raster cells. Based on the distance decay theory, we apply the negative logistic function in an accessibility model. Total amount of passenger flow is obtained by combining population locations with probability of travel to the railway intersection. The parameters, which play a key role in the accessibility model, are tested in an empirical study of Wuhan, China. Through proper parameter configuration, the accessibility model may achieve acceptable accuracy for predicting railway intersection trip production.
\end{abstract}

\section{Introduction}

In the 21st Century, the rapid development of urban railway intersection in Chinese major cities requires scientific planning and design. railway intersection ridership predicting provides important data support for project proposal, planning, design, and operation. First, traffic volume forecast requires the knowledge of spatial distribution of traffic flow and demand. Traditional disaggregate traffic models use smaller zones for spatial analysis which is more suitable at the macro-level. When considering the effects of vehicle use and road network structures within the surrounding area of a transit station, the traditional disaggregate models appear rough results ${ }^{[2]}$. Also the traditional models have certain drawbacks, for example, it is difficult to control the accuracy of results, tedious work for data entry, insensitive to vehicle use patterns, and high operating cost ${ }^{[1]}$. We can use relatively simplified alternatives to forecast the transportation demand on a railway intersection site. A typical alternative is to directly use population data, vehicle use characteristics, and level of public transit service in the surrounding area of a site to predict the potential ridership ${ }^{[3,4]}$. These methods are less time consuming, low cost, and complement to the 'Four Stages Predict Method'.This paper is based on distance decay theory to study the passenger flow around railway intersection sites. To verify the results, we evaluate the direct passenger-flow forecasting process using Wuhan railway intersection Line One as an empirical study.

\section{Accessibility Based Direct railway intersection Passenger-Flow Predicting Model}

\section{Predicting Process}

For a commuter, the accessibility to railway intersection is defined as the ease of travel from an origin to a railway intersection site. Ridership is directly affected by the reachability of a transit site ${ }^{[5,6,7]}$. A large number of domestic and foreign survey data on railway intersection has indicated that distance is the key factor affecting the accessibility of passengers to railway intersection. In the Nethervehicles, Keijer and Rietveld found that people live within the range of 500 1,000 meters from a railway intersection site use $20 \%{ }^{[8]}$ less railway intersection service than those who live less than 500 meters from a railway intersection site. Untermann discovered a distance decay function that most transit users are willing to walk 150 meters to a transit site ${ }^{[9]}$. Cervero concluded that if both the origin and destination are close to the railway intersection, the ridership will increase dramatically ${ }^{[10]}$. In Beijing, along with the increase of distance to a railway intersection site, the share of walk-to-transit decreases fast ${ }^{[11]}$; Survey data from Guangzhou also supports this point of 
view $^{[12]}$. Other studies indicate that functional relationship appears between the use of public transit and distance. Levinson et al. shows that with the increase in walking distance, total number of ridership appears linear reduction ${ }^{[13]}$. A public transit survey conducted by Zhao indicated that with walking distance to the bus station increases, ridership reduces exponentially ${ }^{[14]}$.

An accessibility model can be obtained by constructing a functional relationship between travel impedance and the probability of using public transit. A percentage of population that travel by railway intersection can be obtained by analyzing the residential characteristics using accessibility model. To calculate the probability of public transit usage, residents are divided into small spatial grid cell units; estimated railway intersection ridership for each cell unit is calculated using the total number of population in each cell multiplied by the probability of ridership. Total passenger flow is achieved by summing up estimated railway intersection ridership of each cell unit around a certain transit site. Fig.1 represents a flow chart of the predicting process. The key factors are the accessibility of transit sites and population distribution.

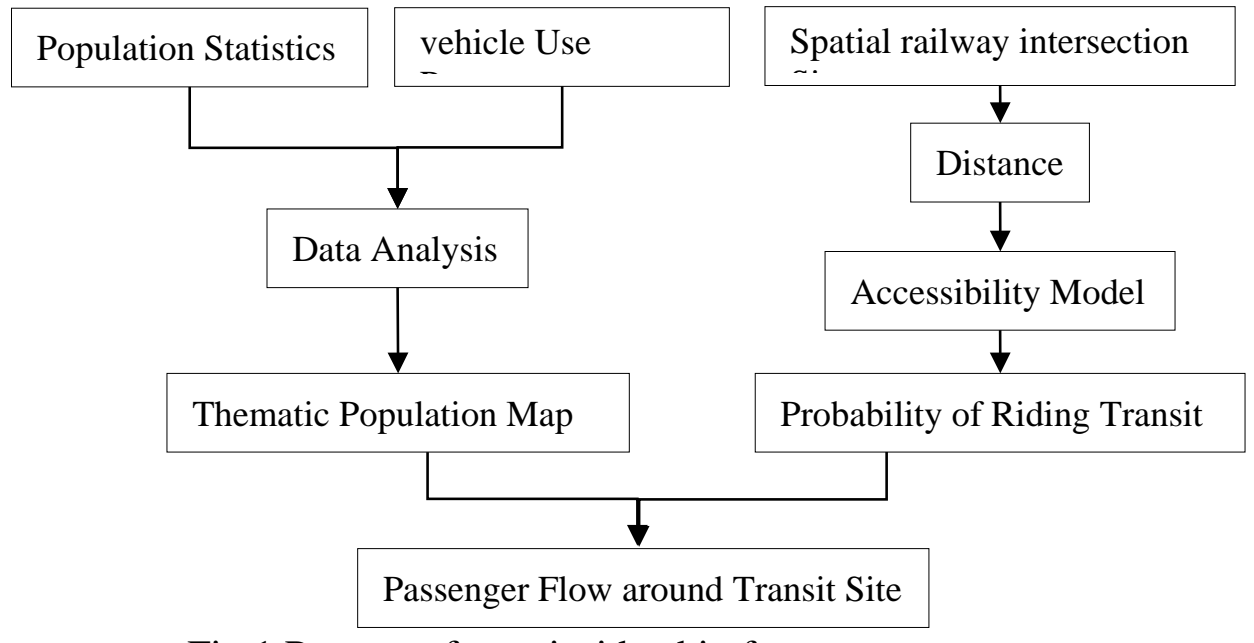

Fig.1 Process of transit ridership forecast

\section{Passenger Flow Forecast under Different Parameters}

At each railway intersection site, passenger flow is predicted based on available data. We use trial algorithm approach to predict the passenger flow at each site, and found that the efficiency of surrounding road network has a greater impact on the site reachability. Because road network characteristics are varied, the accuracy of passenger flow forecast at each site appears uncorrelated. Using the mean error for all sites as a base, we select four cases of parameters with smallest errors for analysis, results are listed in Table 1 :

Table 1 Predict results of different parameters

\begin{tabular}{|l|l|l|l|l|l|}
\hline Station & $\begin{array}{l}\text { Passenger } \\
\text { flow }\end{array}$ & M159 & M155 & $\begin{array}{l}\text { M14 } \\
8\end{array}$ & M147 \\
\hline Tuoluo Kou & 437 & 229 & 478 & 257 & 310 \\
\hline Gutian Yilu & 550 & 181 & 442 & 209 & 258 \\
\hline Gutian Erlu & 1598 & 437 & 1111 & 515 & 651 \\
\hline Gutian Sanlu & 1507 & 515 & 1434 & 618 & 807 \\
\hline Gutian Silu & 1469 & 573 & 1252 & 649 & 791 \\
\hline Hanxi Yilu & 1163 & 786 & 1747 & 896 & 1096 \\
\hline Zongguan & 1701 & 516 & 1246 & 598 & 751 \\
\hline Taipingyang & 575 & 476 & 1351 & 570 & 745 \\
\hline Qiaokou & 1223 & 667 & 1731 & 785 & 997 \\
\hline Chongren Lu & 1840 & 1094 & 2443 & 1245 & 1530 \\
\hline Liji Beilu & 3043 & 634 & 1681 & 756 & 974 \\
\hline Youyi Lu & 1876 & 1302 & 2858 & 1481 & 1809 \\
\hline Xunlimen & 3120 & 1169 & 2992 & 1375 & 1748 \\
\hline
\end{tabular}




\begin{tabular}{|l|l|l|l|l|l|} 
Dazhi Lu & 1700 & 1320 & 3278 & 1536 & 1935 \\
\hline Sanyang Lu & 1533 & 1323 & 3214 & 1537 & 1933 \\
\hline Huangpu Lu & 2466 & 708 & 1868 & 833 & 1066 \\
\hline TouDao Jie & 927 & 580 & 1270 & 653 & 793 \\
\hline Erqi Lu & 1586 & 536 & 1247 & 617 & 764 \\
\hline Xuzhou Xincun & 1196 & 512 & 1104 & 577 & 698 \\
\hline Danshuichi & 907 & 139 & 362 & 164 & 208 \\
\hline Xinrong & 1860 & 167 & 363 & 189 & 227 \\
\hline
\end{tabular}

Use the values of $\mathrm{R}$, which is the error between predicted number of passenger flow and the actual number of passenger flow, as an index to evaluate the accuracy of predicted results.

$$
K(X)=\left\{\left(X_{1}=x_{1}, X_{2}=x_{2}, \cdots, X_{n}=x_{n}\right) \mid \forall c_{i},\right.
$$

Where:

$\mathrm{X}$ represents the predicted number of passenger flow;

$\mathrm{C}$ represents the actual observed number of passenger flow.

Figure 2 shows the error distribution between predicted passenger flow and actual passenger flow. As it shown in illustration, the predicted value is more sensitive to parameter variations. Overall, the errors of sites on the line ends are relatively large, and the errors between adjacent sites are closer; the errors of the central line sites are smaller. The errors' variation trend of program M159, M147, M148 are almost consistent. The average error of M147, which are fluctuations in -0.3 to 0.87 and with an average error of 0.31 , is minimum. The error values of Xingrong station, Danshuichi station, LiJi Beilu Station are large, which are not less than 0.65; the error values of Hanxiyilu station, Youyilu Station, and Toudaojie station are small, which are not greater than 0.05.

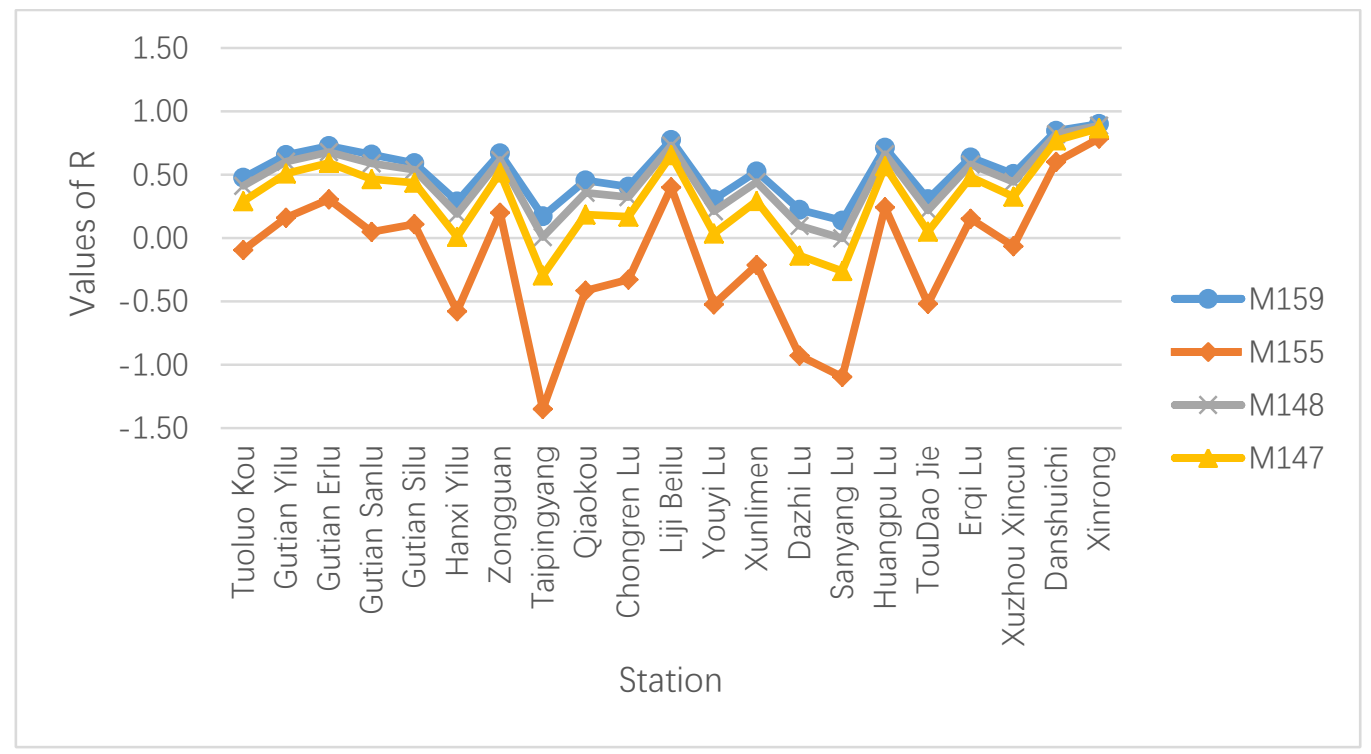

Fig 2 Relative error of passenger flows using four sets of parameters

\section{Conclusion}

Site based accessibility and population distribution can be used to predict passenger flows at railway intersection sites. More accurate prediction parameters can be obtained through comparison between results of different parameter combinations and actual observed results. In addition to traditional forecasting methods, this study provides another more convenient choice for passenger flow forecasting. Even though the configuration of parameters is quite difficult, this method requires less data and a short data analysis cycle. The further improvement on this study is from the three following points. First, use more thorough site survey to get population and road network based distance measures; Second, choose a fitting method of parameters to determine their 
computing methods and empirical values under different vehicle use characteristics; Last, generate relationship analysis between the feeder bus around railway intersection site and distribution of passenger of a site.

\section{References}

[1] Marshall N, B Grady. Sketch transit modeling based on 2000 census data[C]. Transportation Research Record: Journal of the Transportation Research Board,2006,:-

[2] Chu X. Ridership models at the stop level[R]. National Center for Transit Research, University of South Florida, Tampa. 2004

[3] Cervero R. Alternative approaches to modeling the travel-demand impacts of smart growth[J]. Journal of the American Planning Association, 2006, 72(3): 285-295

[4] Chen B Z, Shen Y Z, Wang Z Q, Zhang J. railway intersection Passenger Forecasting: a theoretical study in Shanghai[J]. Urban Transport Of China, 2009, 7(1):13-19

[5] Chien S I, QIN Z Q. Optimization of bus stop locations for improving transit accessibility[J]. Transportation planning and Technology, 2004,27(3):211-227

[6] Murray, A.T. and X. Wu, Accessibility tradeoffs in public transit planning. Journal of Geographical Systems, 2003. 5(1): p. 93-107.

[7] Bertolini L. Spatial development patterns and public transport: The application of an analytical model in the Nethervehicles. [J].Planning Practice and Research, 1999,14(2):199-210

[8] Keijer M J N, Rietveld P. How do people get to the railway station? The Dutch experience. [J].Transportation planning and Technology,2000, 23(3):215-235 